\title{
SLEEP ARCHITECTURE IS RELATED TO THE SEASON OF PSG RECORDING IN 8-MONTH-OLD INFANTS
}

Anja Kärki ${ }^{\mathrm{a}}$ E. Juulia Paavonen ${ }^{\mathrm{b}, c}$, Anna-Liisa Satomaa ${ }^{\mathrm{a}}$, Outi Saarenpää-Heikkiläd,e, Sari-Leena Himanen ${ }^{\mathrm{a}, \mathrm{e}}$

a) Department of Clinical Neurophysiology, Tampere University Hospital, Medical Imaging Centre and Hospital Pharmacy, Pirkanmaa Hospital District, Tampere, Finland;

b) Pediatric Research Center, Child Psychiatry, University of Helsinki and Helsinki University Hospital, Helsinki, Finland;

c) Department of Public Health Solutions, National Institute for Health and Welfare, Helsinki, Finland;

d) Center for Child Health Research Tampere University and Tampere University Hospital, Tampere, Finland;

e) Faculty of Medicine and Health Technology, Tampere University, Tampere, Finland

*Corresponding author:

Anja Kärki, MD

Department of Clinical Neurophysiology,

Tampere University Hospital, Medical Imaging Centre and Hospital Pharmacy,

Pirkanmaa Hospital District,

PO BOX 2000, FI 33521

Tampere, Finland

Tel.: +358 3 31165095; Fax: +358331164199.

E-mail address: anja.karki@tuni.fi 


\section{Abstract}

To date, little is known about the impact of season on infant sleep. In higher latitudes, the duration of daily light time varies substantially between different seasons, and environmental light is one potential factor affecting sleep. In this cohort study, one-night polysomnography (PSG) was performed on 72 healthy 8-month-old infants in 2012 and 2013 to study the effect of season on the sleep architecture of young infants in Finland. The children were divided into four subgroups, according to the amount of light during their birth season and the amount of light during the season of the PSG recordings, corresponding to spring, summer, autumn, and winter. We found that the season of birth did not have an impact on the infants' sleep architecture at 8 months of age, but the season of the PSG recording did have an effect on several sleep variables. In the PSGs conducted during the spring, there was less N3 sleep and more N2 sleep than in the PSGs conducted during the autumn. In addition, there was more fragmented sleep during spring than autumn. According to our data, the season has an effect on the sleep architecture of young infants and should therefore be considered when evaluating the PSG findings of young infants. The exact mechanisms behind this novel finding remain unclear, however. The findings imply that infants`sleep is affected by the season or light environment, as is the case in adult sleep. Since potential explanatory factors, such as direct natural or artificial light exposure and the melatonin levels of the infants, were not controlled, more research is needed in future to better understand this phenomenon.

\section{Keywords}

Infant sleep, polysomnography, season, chronobiology, seasonality, environmental light, SWS, N3 sleep. 


\section{Introduction}

The human circadian clock is entrained via different environmental factors, the most powerful of which is light. The season of the year has an impact on the amount of environmental light, especially in the Arctic and Antarctic regions. Previous studies have demonstrated that seasons can affect sleep quality and quantity in adults. Moreover, according to the findings of an actigraphy study conducted by Paul and co-workers, adults living in the Arctic environment sleep longer during the dark winter months than during the light summer months (Paul et al. 2015). Similar findings have been reported among preindustrial African and South American societies by Yetish and co-workers (Yetish et al. 2015). Moreover, a recent review demonstrated that adults have lower subjective sleep quality, more sleep fragmentation, and less deep sleep during the Antarctic winter (Pattyn et al. 2018). Conversely, an abundance of REM/R or active sleep has been observed in adults during the summer months in the Antarctic and in an actigraphy study of 7-month-old infants in Mediterranean countries (Cohen et al. 2012; Pattyn et al. 2017). Data from one questionnairebased study reported that infants born in spring sleep for the longest during the night (Iwata et al. 2017). In addition, another recent study showed that infants born in spring are less vulnerable to sleep-wake-rhythm challenges at the age of 3 months (Paavonen et al. 2019).

Dim, artificial light can also affect sleep architecture. Exposure to artificial light at night is found to decrease total sleep time and sleep efficiency and to increase sleep fragmentation and REM sleep, at least in adults (Cho et al. 2016, 2018). In addition, evening light exposure at 6500K has been found to reduce frontal slow-wave activity in the first sleep cycle (Chellappa et al. 2013).

In our previous PSG study, we found that 1-month-old infants born in the summer in Finland present more R sleep than infants born in winter (Kärki et al. 2019). In addition, infants presented 
less T sleep (transient, immature sleep stage) during the summer when compared to the winter, and shorter nighttime sleep when compared to the autumn.

The environmental light circumstances of Finland are extraordinary and vary depending on latitude and the season of the year. The city of Tampere is located in southern Finland, and the daily amount of light varies significantly in the Tampere region, ranging from a minimum of $5.3 \mathrm{~h}$ during the shortest days in winter to a maximum of $19.5 \mathrm{~h}$ during the longest summer days. When considering this large variation in seasonal light, we hypothesized that it has an effect on sleep architecture. It is, however, possible that some of the findings of our previous PSG study could be explained by the light season of the year during which the PSG was performed and not by the birth season itself. The aim of this present study is to investigate whether our previous findings concerning the abundance of R sleep (REM sleep) in infants born during the summer is still present in the same cohort 7 months later. And if not, we aim to find out how the season affects the REM/R sleep and sleep architecture of 8-month-old infants in general.

\section{Materials and methods}

Our work is part of a larger study (CHILD-SLEEP). CHILD-SLEEP is a multidisciplinary project aiming to evaluate various aspects of sleep in infancy and early childhood. The study process has been described previously in detail (Paavonen et al. 2017; Satomaa et al. 2015). The present study was conducted on a subgroup of this cohort that comprised infants who underwent an ambulatory overnight PSG at the age of 8 months.

In short, the families were asked to participate in the larger study prenatally at their maternity clinics. Postnatally, all the families who fulfilled the inclusion and exclusion criteria were requested, while at the maternity ward, to participate in the polysomnography (PSG) substudy. The inclusion criteria for the PSG-study were healthy, full-term, and uneventful birth (conceptional age 
38 weeks or more), Apgar score $\geq 8$ at 1 minute, and birth weight $\geq 2500 \mathrm{~g}$. In addition, the mothers had to be medication-free (from drugs acting on the central nervous system) during pregnancy. Ultimately, 76 infants who were born at the conceptional age of 38-42 weeks participated in the PSG study at the age of approximately 8 months (median 7.8 months, range 7.4-9.0 months). The PSGs were performed at the homes of the families in the Tampere region in 2012 and 2013. The same PSG technician and medical physicists acquired all the recordings. The parents were asked to keep notes of any events (e.g., feeding and diaper changing) during the night.

In addition, we collected information regarding the infants’ current nutrition, growth, sleeping habits (regularity of bedtime routines, typical time of falling asleep), and the number and duration of daytime naps. We obtained this information via a questionnaire and from the child health clinics' data. In addition, data on the nighttime light levels in bedrooms was gathered via questionnaire at the 3-month time point. The data were processed into the following categorical variables: nutrition: solid food vs only milk; breastmilk only vs breastmilk and infant formula or infant formula only; bed time routines: always regular vs mostly regular vs irregular; typical time of falling asleep: expressed in time (XX h XX min) in 30 minute epochs; number of daytime naps: one, two, three, or more per day. The data regarding bedroom light level during the night at 3 months of age was dichotomized as follows: totally or mostly dark vs dim or quite light. Information regarding the infants' growth and daytime sleep duration data were also gathered via a questionnaire and the child health clinics’ data, and they were processed into continuous variables. There were seven answers missing in the questionnaires regarding solid nutrition, and six regarding milk vs infant formula consumption, six regarding bedtime routines and typical time of falling asleep, and seven regarding the number of daytime naps. There were three missing answers regarding the bedroom light conditions at 3 months of age and 12 missing answers regarding weight, 18 regarding weight-forlength/height Z-score, and six regarding daytime sleep duration. 
The study has been approved by the local ethical committees. All the recruited parents gave their written informed consent. Where applicable, the ethical standards of biological rhythm research instructions were followed (Portaluppi et al. 2010).

\section{Recordings and visual analysis}

From the 76 recordings of 8-month-old infants, 4 were lost due to technical failure. In total, 72 infants (38 girls, 34 boys) were included in the study. The ambulatory PSGs were obtained using the Embla Titanium system. The following signals were recorded: 6 channels of electroencephalography (EEG) (F4-A1, C4-A1, O2-A1, F3-A2, C3-A2, O1-A2), right and left electro-oculography, submental EMG, oxygen saturation (pulse oximeter, Nonin), thoracoabdominal inductance plethysmography, diaphragmatic and abdominal EMG, Emfit mattress sensor, and ECG. Airflow was measured by oronasal thermistor (Dymedix). In addition, a snoring sensor (piezo) was used. To minimize the potential disturbance to sleep of the recording equipment, the nasal pressure transducer was omitted from the protocol (Goodwin et al. 2001).

The PSGs were scored into sleep stages in 30s epochs with Somnologica Studio 5.0 software by two independent, experienced clinical neurophysiologists (ALS, SLH) with an agreement of 82.3\% (range, 69.5 to $91.1 \%$ ) and kappa score of 0.77 (range, 0.61 to 0.88 ) indicating substantial agreement (Landis and Koch 1977). The sleep stages, respiratory events, and arousals were visually scored according to the current pediatric guidelines (Berry et al. 2012). The differences in scoring were discussed and consensus scoring was established for further analyses. In short, the EEG was scored as Stage REM, Stage N1, N2, N3, or wakefulness (W) according to the rules of the AASM 2012 manual. The duration of each sleep state divided by total sleep time (TST) was expressed as REM\%, N1\%, N2\%, and N3\%, with the last three forming NREM\%. An arousal with a duration of $\geq 30$ s or an arousal followed by a wake epoch was equaled as an awakening. The number of awakenings and arousals divided by TST were summed together to form the sum of awakening and 
arousal index (AWARI). Since the recordings were ambulatory home-recordings, a video was not recorded.

\section{Statistical analysis}

Cut-off points for seasonal light were adjusted according to a yearly sun graph for the Tampere region (Time and Date AS 2019). The cut-off points were set to the beginning of February, May, August, and November. This allowed us to separate four equally long periods with a daily light variation of approximately 5-19.5 h: the period of dark (group D, Nov 7-Feb 4) with 5.3-8 h of daily light, the period of increasing light (group IL, Feb 5-May 5) with 8-16.5 h of daily light, the period of light (group L, May 6-Aug 5) with 16.5-19.5 h of daily light, and the period of increasing darkness (group ID, Aug 6-Nov 6) with 16.8-8 h of daily light. The infant sleep data obtained by the PSG recordings were analyzed according to the four seasons defined above, both according to birth season and PSG recording season.

Normality of data was assessed by visual inspection and statistical tests. In the whole cohort of 8month-olds, all the studied variables apart from sleep latency and age at PSG-recording time were Gaussian. In the birth season groups, Gaussian variables were TIB, TST, N2\%, N3\%, number of arousals and awakenings, arousal and awakening index, sum of awakening and arousal index (AWARI), growth information (weight and weight-for-length/height Z-score), and daytime sleep duration. Non-Gaussian variables were N1\%, NREM\%, REM\%, wake after sleep onset (WASO), sleep efficiency index, sleep latency, REM-latency, and age at PSG-recording time. In addition, sleep latency and age at PSG-recording time had different variances. Gaussian variables of PSG season groups were TIB, TST, N2\%, N3\%, REM\%, wake after sleep onset (WASO), sleep efficiency index, number of arousals and awakenings, arousal and awakening index, sum of awakening and arousal index (AWARI), growth information (weight and weight-for-length/height Z-score), and daytime sleep duration. Non-Gaussian variables were N1\%, NREM\%, sleep latency, 
REM-latency, and age at PSG-recording time. In addition, N2, N3, sleep latency, and age at PSGrecording time were variables with different variances. In the analysis of PSG recordings conducted within two weeks after the start of daylight-saving time, the non-Gaussian variables were N1, arousal index, and AWARI.

To evaluate the individual changes in sleep parameters between 1 and 8 months of age, we formed novel variables, that is, the change\% of all the sleep parameters of interest between the 1- and 8month PSG recordings. For these variables, the following formula was used to calculate individual change\% for each of the sleep parameters: (X 8 mo - X 1 mo)/ X 1 mo x 100\%, with X representing each sleep variable of interest. The start times of PSG recordings at both PSG time points (1 and 8 months) were non-Gaussian.

Gaussian variables were analyzed using one-way analysis of variance, whereas non-Gaussian variables or variables with different variances were analyzed with the Kruskal-Wallis test. Post-hoc tests were performed using Tukey’s test and Dunn-Bonferroni’s test, respectively. Categorical variables were analyzed using the Chi-square test. Two related variables were analyzed using the Wilcoxon test. Two independent variables were analyzed using the Mann-Whitney-U-test.

Generalized linear models were computed to study the effect of season of birth and season of PSG recording while controlling for sex and age. All sleep variables were assessed in separate models. Statistical analysis was performed using SPSS version 22.

\section{Results:}

\section{Sleep parameters}

The sleep parameters with demographic data of the 72 children at 8 months of age are presented in Table 1 . In total, $65.2 \%$ of parents reported infants consuming only breastmilk, $18.2 \%$ only infant formula, and $16.7 \%$ both breastmilk and infant formula. In addition, $72.5 \%$ families answered that 
the bedroom was totally or almost dark, whereas $27.5 \%$ answered the bedroom was dim or quite light. A further 26.1\% had blackout curtains and 27.5\% reported using nighttime artificial light.

\section{Table 1}

\section{Sleep parameter evolution between 1- and 8-month-old infants}

There were 69 infants recorded both at the age of 1 and 8 months. The start times of the 8-month and 1-month-old infants’ PSG recordings differed from each other, with the 8-month recordings starting approximately at 19:53h (range, 18:15h.-21:45h) and the 1-month recordings starting approximately at 21:15h (range, 19:15h-23:00h) ( $\mathrm{p}<0.001)$. There was clear evolution in all the sleep parameters when the 1-month recordings were compared to the 8-month recordings - there was an increment in TST, SEI\%, and N-sleep/NREM\% $(\mathrm{p} \leq 0.001)$. A decrement was observed in TIB, wake after sleep onset (WASO), R-sleep/REM\%, the number of awakenings, and the number of arousals and corresponding indexes (p values ranging from $<0.001$ to 0.020 ). The change percentages of some of these sleep parameters are presented in Figure 1.

\section{Figure 1}

\section{Birth seasonal groups at 8 months of age}

Among 72 infants, there were 11 (15.3\%) infants born during the spring (Feb 5-May 5, increasing light, group IL), 11 (15.3\%) born during the summer (May 6-Aug 5, light, group L), 36 (50.0\%) born during the autumn (Aug 6-Nov 6, increasing dark, group ID), and 14 (19.4\%) were born during the winter (Nov 7-Feb 4, dark, group D). Data regarding these groups are presented in Table 2. 
There were no significant gender differences in the four birth season groups ( $\mathrm{p}$ value 0.276 ). The start time of the PSG recordings did not differ among the birth season groups $(p=0.262)$. The groups were diverse regarding the infant's age at the time of the recording $(p=0.007)$. However, the differences were very small (7.4-9.0 months), and the vast majority of recordings were conducted at 7 or 8 months of age $(n=71)$.

There was no difference in nutrition between the groups, with all parents reporting that the infants ate solid food in addition to milk. The type of milk consumed did not differ between the groups $(\mathrm{p}=0.614)$. There were no differences in weight at 8 -month age $(\mathrm{p}=0.449)$ or weight-forlength/height Z-score ( $\mathrm{p}=0.591$ ) between the groups. There were also no significant differences in the number of naps ( $\mathrm{p}=0.251$ ), with the vast majority (63 infants) having two or three or more naps per day. The reported duration of daytime sleep (hours) did not differ between the groups either ( $\mathrm{p}=0.241$ ), with the mean daytime sleep duration being $3.5 \mathrm{~h} /$ day. No differences were reported in the regularity of bedtime routines between the groups ( $\mathrm{p}=0.992)$, nor differences in the typical time of falling asleep (mean 20:49h, SD 0.06, p=0.648), with the majority of infants (57 out of 66 answers) typically falling asleep between 19:00h and 21:30h. There was no difference in bedroom light conditions between the groups at 3 months of age $(p=0.540)$.

The percentage of N2 differed across the four birth season groups $(\mathrm{p}=0.035)$ and, according to a post hoc test, $\mathrm{N} 2 \%$ was higher in group $\mathrm{L}$ than in group $\mathrm{D}(\mathrm{p}=0.027)$. Moreover, the percentage of N3 differed among the four groups ( $\mathrm{p}=0.028)$, with the post hoc test showing the percentage of N3 being lowest in group L compared to group $\mathrm{D}(\mathrm{p}=0.018)$. The percentages of NREM sleep and REM sleep did not differ between the four season groups (p values being 0.323 and 0.278 , respectively).

When analyzing the relations between individual birth and PSG recording season, there were 14 infants born during the winter (group D), and 11 (78.6\%) of their PSGs were recorded during the 
following autumn (group ID). There were 11 infants born during the summer (group L) and 9 (81.8\%) of their PSGs were recorded during the following spring (group IL).

In the generalized linear model, when controlling the results regarding birth season for age and sex, the results regarding N2\% and N3\% did not remain statistically significant (Table 2, adjusted pvalues 0.135 and 0.104 , respectively).

\section{$\underline{\text { Table } 2}$}

\section{Recording seasonal groups at 8 months of age}

Among the 72 infants recorded at 8 months of age, 24 (33.3\%) recordings were performed in the spring (Feb 5-May 5, increasing light, group IL), 24 (33.3\%) performed in the summer (May 6Aug 5, light, group L), 15 (20.8\%) performed in the autumn (Aug 6-Nov 6, increasing dark, group ID), and 9 (12.5\%) performed in the winter (Nov 7-Feb 4, dark, group D). Data concerning these groups are presented in Table 3.

There was no significant difference in the gender or age of the infants in the four PSG recording groups (p-values 0.645 and 0.117 , respectively). The start time of the PSG recording did not differ among the four groups $(\mathrm{p}=0.266)$.

All the infants consumed both milk and solid food. The type of milk consumed did not differ between the groups $(p=0.688)$. At 8 months, there was neither a difference in weight $(p=0.955)$ nor a difference in weight-for-length/height $\mathrm{Z}$-score $(\mathrm{p}=0.889)$ between the groups. There was also no significant difference in the number of daytime naps $(\mathrm{p}=0.750)$, with the vast majority (63 infants) having two or three or more naps per day. The reported duration of daytime sleep (hours) did not differ significantly between the groups either $(\mathrm{p}=0.372)$, with the mean daytime sleep duration being $3.5 \mathrm{~h} /$ day. There was no difference in the regularity of bedtime routines between the groups 
$(p=0.666)$, nor was there a difference in the typical time of falling asleep (mean 20:49h, SD 0.06, $\mathrm{p}=0.671$ ), with the majority of infants (57 out of 66 answers) typically falling asleep between 19:00h and 21:30h. There was no difference in bedroom light conditions between the groups at 3 months of age $(p=0.767)$.

The percentage of $\mathrm{N} 2$ differed among the groups $(\mathrm{p}=0.003)$, and in the post hoc test $\mathrm{N} 2$ was higher in group IL than in group ID ( $\mathrm{p}=0.001)$. The percentage of N3 differed among the groups $(p=0.006)$, and in the post hoc test N3 was lower in group IL than group ID ( $p=0.003)$. The number of minutes spent awake during the night (WASO) differed among the groups ( $\mathrm{p}=0.002$ ), and in the post hoc test WASO was lower in group L than IL and ID ( $p=0.034$ and 0.008 , respectively) and lower in group $\mathrm{D}$ than in ID ( $\mathrm{p}=0.035$ ). The sum of awakening and arousal indexes (AWARI) differed among the groups ( $\mathrm{p}=0.012$ ), with the post hoc test showing AWARI being higher in group IL than in group ID $(\mathrm{p}=0.019)$.

\section{$\underline{\text { Table } 3}$}

\section{Effect of changing to daylight saving time}

In group IL, 11 (45.8\%) PSGs were recorded within two weeks after the vernal equinox and 7 (29.1\%) recorded within two weeks after the start of daylight saving time. When comparing the sleep parameters of these 7 infants (the post-daylight saving time group: those for whom PSG recordings were taken shortly after the start of daylight saving time) with the rest of the cohort, the post daylight saving time group presented more N1-sleep (median 8.0\%, range 3.1-18.0\%) than the rest of the cohort (median 5.8\%, range 1.9-18.0\%), (p-value 0.046). In addition, the post daylight saving time group had a higher arousal index (median 11/h, range 5.2-14.4/h) than the rest of the 
cohort (median 7.3/h, range 3.2-13.3/h), (p-value 0.037) and higher AWARI (median 13.8/h, range 9.9-17.8/h vs. 10.1/h, 5.2-16.5/h, p-value 0.013). During the autumn (group ID), there were two (13.3\%) PSGs recorded within two weeks after the autumnal equinox and one (6.7\%) was recorded within two weeks after the start of standard time.

\section{Generalized linear models}

In the generalized linear models, we controlled the results regarding PSG recording season for age and sex. In these analyses, the results regarding N2, N3, WASO, and arousal and awakening indexes remained statistically significant (adjusted p-values $0.001-0.042$, Table 3). None of these variables were dependent on age or sex.

\section{Discussion}

In our previous study, we found a greater abundance of R/REM sleep in 1-month-old infants born during the summertime than those infants born in the winter (Kärki et al. 2019). In our current study, when the children are approximately 7 months older, however, we did not find any REM sleep differences between the birth season groups. Instead, we found winter-born infants (group D) presented more N3 sleep and less N2 sleep than summer-born infants (group L), but these findings did not remain in the generalized linear model. PSG recordings were conducted between 7 and 9 months after birth, and it seems that the PSG recording season might have contributed to the previously mentioned N3 and N2 sleep findings. For birth season group L, most of the PSG recordings at 8 months of age were conducted during the following spring (IL), and for birth season group D most of the recordings were conducted during the following autumn (ID).

Indeed, we found several differences in sleep architecture when we grouped the infants according to the season when the 8-month PSG was conducted. In the recordings conducted during the springtime (group IL), the infants presented more N2 and less N3 sleep when compared to 
recordings conducted during the autumn (group ID). In addition, sleep was more fragmented in group IL than in group ID. Moreover, group IL presented more wake after sleep onset than group L. In this manner, our PSG-based data suggest that at the age of 8 months the recording season has a greater effect on sleep parameters than birth season, and the season of the PSG recording might be a potential explanatory factor behind certain differences in sleep architecture in 8-month-old infants. The results concerning the PSG recording seasons remained when analyzed in the generalized linear model.

According to previous studies, there have been some season-dependent sleep architecture findings. To date, evidence suggests that the seasonal effect increases the time spent in bed and the sleep length during winter (O'Connell et al. 2014; Paul et al. 2015). In children, summer is found to provoke more restless sleep and active sleep at the expense of quiet sleep (Aronen et al. 2002; Cohen et al. 2012). However, to the best of our knowledge, there is a scarcity of evidence regarding the seasonal effects on the sleep quality and architecture of infants. Moreover, the abovementioned studies were not based on PSG data.

The mechanism behind our season-dependent PSG findings is unclear. However, it is possible that the rapidly changing day and evening light conditions typically found in spring affect infant sleep by modifying circadian regulation. Indeed, long bright days induce different plastic changes in the SCN network than shorter days (Rohr et al. 2019), and these changes in plasticity might affect sleep architecture. There is also evidence that sleep architecture is related to daytime light exposure in young adults (Wams et al. 2017). Especially, exposure to early afternoon light seems to be beneficial, improving the sleep of infants (Harrison 2004). The Finnish custom of taking babies out for a walk and letting the babies sleep outside in a stroller exposes them to natural light conditions regardless of season. This may have an effect on the total daytime natural light exposure and the subsequent sleep as well. However, we do not have accurate data on the natural or artificial light exposure of infants during the day. 
Conversely, blue light exposure during the evening is harmful for sleep (Chellappa et al. 2013). Thus, the rapid increase in natural evening light during spring might explain the paucity of N3 that we found, if the assumption is made that natural light during the late evening can affect sleep in a similar way than artificial light does. In our study, N3 was higher during the autumn than during the spring. In the autumn, the amount of natural evening light is reduced, but afternoon light is preserved, which can potentially explain the better sleep quality in our ID group, whereas during the spring the abrupt increase in light, also in the evenings, might have reduced deep sleep in group IL.

In adults, however, a major decrease in the amount of N3 was detected during the Antarctic summer, which typically has excessive light (Pattyn et al. 2017). Therefore, an unexpected finding was that N3 sleep did not diminish significantly in group L, even though the summer evenings in Finland are even brighter than the evenings in spring. If our finding of the relative paucity of N3 sleep during springtime is due only to natural light, one might expect that there would also be similar findings in group L. We can only speculate on the reasons behind this finding. During springtime, the amount of light increases very rapidly in Finland and infant sleep is affected by natural light, but the infants start adapting to the light conditions during the end of spring and the beginning of summer. Groups IL and L consisted of the same number of children, so a lack of statistical power is unlikely to explain our N3 sleep finding. In addition, both the vernal equinox and the change to daylight saving time increase the amount of evening light very rapidly after the end of March. It is therefore possible that the adaptation mechanisms cannot respond quickly enough. In group IL, approximately one-third of the PSG recordings were conducted two weeks after the change to daylight saving time and almost half of the recordings were conducted after the vernal equinox, both of which can affect the results via an altered exposure to light and a change in the daily routines or circadian rhythm. Soon after the change to daylight saving time, infants tended to present more light and fragmented sleep when compared to the rest of the cohort. We can only 
speculate on the reasons behind this novel finding. Babies live according to the parental rhythm, which is obviously affected by the change to daylight saving time. Perhaps, the schedules and bedtime routines of families are changed because one hour is lost from the day.

In the Tampere region, people are exposed to considerably more natural light during the summer than during the darkest wintertime (max. $19.5 \mathrm{~h}$ vs min. $5.3 \mathrm{~h}$ ). Therefore, the sleep hormone melatonin, which is secreted during the dark night, might explain some of the seasonal sleep parameter differences that we found in the present study. This is supported by the study of Leu and co-workers in the United States who reported that increased melatonin levels in autistic children were associated with higher amounts of N3 sleep and less N2 sleep (Leu et al. 2011). In contrast to our study, they showed that season alone was not associated with N2 or N3 sleep.

According to a previous Finnish study, season has an impact on melatonin concentration since higher levels of nighttime melatonin were observed during winter (Paakkonen et al. 2008). Contrary to the findings of that study, another group found that, at 8 weeks of age, infants born in June secrete more melatonin during the nighttime than children born in December (Sivan et al. 2001). It seems therefore possible that other factors that affect melatonin secretion and sleep quality might exist since previous findings of melatonin secretion and season are contradictory.

Indeed, melatonin secretion can be modified by artificial light (Burgess and Molina 2014; Higuchi et al. 2014). For example, artificial light in the bedroom during the evening and night suppressed melatonin levels (Gooley et al. 2011). In addition, light exposure seems to have a greater impact on children in suppressing melatonin production (Higuchi et al. 2014). When considering these findings to ours, rapidly increasing evening light during the spring could potentially suppress melatonin secretion, which could, in turn, have reduced the amount of N3 in group IL, whereas the darker evenings of the autumn would have increased N3 sleep via increased melatonin levels. 
Unfortunately, melatonin levels were not controlled in our study, and thus these questions remain unanswered.

In Finnish households, artificial light during the darker seasons, autumn and winter, is often substantial during the daytime and evening, but nighttime natural light, and in most of the families of our cohort, artificial light as well, are absent. It is noteworthy, however, that during the Finnish spring and summer, substantial evening and nocturnal natural light exposure is often hard to control or reduce efficiently due to the evening and midnight sun. Most of the families in our cohort did not use blackout curtains in the bedroom. Therefore, rapidly increasing natural evening and nocturnal light during the Finnish spring can be a potential explanatory factor behind our findings, when compared to daytime light exposure, since people in general can expose themselves to a substantial amount of daytime artificial light regardless of the season.

\section{Findings of whole cohort and evolution of sleep between the ages of 1 and 8 months}

When considering the whole 8-month cohort, there was a lot of individual variation in all of the sleep parameters. In particular, a high variation was found in total sleep time, time in bed, and wake after sleep onset. In our cohort, 8-month old infants presented approximately 66\% NREM and 33\% REM sleep, which resembles previous PSG data of 9-month-old infants (Jenni et al 2004; Louis et al. 1997). The mean of total sleep time during the night was more than $9 \mathrm{~h}$, which is somewhat in line with previous PSG studies (Anders and Keener 1985; Louis et al. 1997; Montemitro et al. 2008).

In addition, many changes were observed when analyzing the evolution of the sleep of infants between 1 and 8 months of age. Total sleep time, percentage of NREM sleep, and sleep efficiency increased, whereas wake after sleep onset, arousals, and amount of R/REM sleep decreased. These findings are in line with previous studies (Duenas-Meza et al. 2015; Jenni et al. 2004; Louis et al. 1997; Montemitro et al. 2008). 


\section{Limitations}

Our data have some limitations. The study population consists of families who volunteered to participate in the CHILD-SLEEP study, which may have had an effect on the cohort. The families participating in the study were typically highly educated (Paavonen et al. 2017). Thus, it is possible that our cohort includes families or infants with more or less sleep complaints than the general Finnish population. Therefore, any direct conclusions regarding the generalization of our findings must be made with caution.

A further limitation of this study is the lack of direct information regarding daytime naps on the day before the PSG. However, the information of typical daytime napping was gathered via a questionnaire and showed no differences in the length of typical daytime sleep nor any differences in the number of naps. In our cohort, both of these daytime sleep variables resembled previous findings (Sadeh et al. 2009; Teng et al. 2012).

It is known that regular and early lights-off times are beneficial for infant sleep (Iwata et al. 2017). There were no differences between our subgroups in the regularity of the bedtime routines or typical times of falling asleep reported by parents. Furthermore, there were no differences between the PSG start times or sleep latencies between the groups, so it is unlikely that large deviations in bedtimes would explain our results.

Unfortunately, we lack information regarding the light conditions in the infants' bedrooms during the PSG night. In the questionnaire data of the same cohort at 3 months of age, most of the parents reported having a totally or almost dark bedroom during the night with no differences between the seasonal subgroups in the level of bedroom light or the use of artificial light. Unfortunately, this information was not gathered at the 8-month time point, but it is likely that the families sleeping routines were similar over the months. We do not have data on possible screen light exposure, but when considering the age of the infants, it is usually not relevant. Even though the infants`expected 
natural light exposures were categorized into four seasonal groups, we cannot provide individual information on the actual or accurate daily or nocturnal light exposure. As previously stated, natural and artificial light during the evening and night may influence sleep. In addition, we lack systematic information regarding feeding during the PSG night. According to previous data, breastfeeding can cause more frequent awakenings during the night in PSG studies conducted at home (Eaton-Evans and Dugdale 1988). Our PSG groups were similar concerning nutrition. All the infants consumed both milk and solid food and there were no differences between groups in the consumption of breastmilk or infant formula. It seems therefore that nutritional differences, such as maternal melatonin in breastmilk, do not explain our results.

\section{Benefits of the study}

So far, the normative data on infant sleep parameters at around 8 months of age have been scarce and widely based on questionnaire or subjective data (Galland et al. 2012; Iglowstein et al. 2003; Sadeh et al. 2009; Teng et al. 2012; Touchette et al. 2013), with only few previously conducted PSG studies (Anders and Keener 1985; Louis et al. 1997; Montemitro et al. 2008). Knowledge of the wide variation of objective sleep parameters in normal infants is valuable when assessing sleep disorders. Our data present infant sleep variables according to the PSG recording season, and understanding the environmental factors that can affect the development of infant sleep quality will help develop more effective preventive interventions in future. To date, less emphasis has been given to the environmental factors, such as seasonal or artificial light, that may play a role in infant sleep quality and architecture.

\section{Conclusions}

In conclusion, according to our PSG data, there is individual variation in infant sleep architecture and sleep quality at the age of 8 months, of which part seems to be explained by the season of the 
year. This is the first study to find that the PSG recording season has an impact on infant sleep parameters in the northern latitudes. Our most important finding was the relative paucity of N3 sleep in the PSG recordings conducted during the spring compared to the autumn. In addition, according to our data, daylight saving time might provoke lighter and more fragmented sleep in infants. This can potentially be due to parental influence, but also to altered evening and nocturnal light exposure during the spring and the subsequent change in daily schedules. Based on previous evidence, it seems that light exposure during the morning and early afternoon is beneficial for sleep, whereas excessive evening and nighttime light exposure is harmful for sleep.

According to our data, infant sleep can be affected by seasonal light exposure, at least in northern latitudes. The rapid increment of evening light conditions during the spring can be one potential explanatory factor behind our findings. There was no deep sleep decrement or sleep fragmentation in the PSGs recorded during the summer, which can be due to the infants' habituation to the light environment. However, due to the limitations of this study, the mechanisms behind our findings remain unclear and more research is needed to evaluate the potential explanatory factors behind the seasonal variations in PSG-findings. In future studies, it would be beneficial to combine actigraphy data, melatonin measurements, and information on the actual light exposure during the day and night.

We are proposing, at least when it comes to young infants, that the effect of the season seems to be a factor to consider when assessing PSG reference values. It is also noteworthy that infant sleep seems to be affected by season as is the case with adult sleep. Therefore, infants should not be forgotten when educating families on sleep hygiene. 


\section{Acknowledgments}

We thank Tytti Koskelo, who organized and performed the PSG recordings.

\section{Funding details}

This study was funded by Academy of Finland, Grant no. 134880 and 308588, by the Competitive Research Financing of the Expert Responsibility area of Tampere University Hospital, Grant nos. 9R007, 9R004, 9S007, 9S058 and 9P013, by Tampere University Hospital Support Foundation, Tampere University Hospital (Grant no MK243), and by Tampere Tuberculosis Foundation (Grant no MS860). AS has received grants from the Finnish Sleep Research Society and The Research Foundation of Pulmonary Diseases.

\section{Disclosure of interest}

The authors report no conflict of interest.

\section{References}

Anders TF, Keener M. 1985. Developmental course of nighttime sleep-wake patterns in full-term and premature infants during the first year of life. I. Sleep. 8(3):173-192.

Aronen E, Fjällberg M, Paavonen E, Soininen M. 2002. Day length associates with activity level in children living at 60 degrees north. Child Psychiatry Hum Dev. 32(3):217-226.

Berry RB, Brooks R, Gamaldo CE, Harding SM, Marcus CL, Vaughn BV. 2012. The AASM manual for the scoring of sleep and associated events: Rules, terminology and technical specifications, version 2.0. Darien (IL/USA): American Academy of Sleep Medicine. [Accessed 2019 Aug 1.]. https://aasm.org/clinical-resources/scoring-manual/ 
Burgess HJ, Molina TA. 2014. Home lighting before usual bedtime impacts circadian timing: A field study. Photochem Photobiol. 90(3):723-726.

Chellappa SL, Steiner R, Oelhafen P, Lang D, Gotz T, Krebs J, Cajochen C. 2013. Acute exposure to evening blue-enriched light impacts on human sleep. J Sleep Res. 22(5):573-580.

Cho CH, Lee HJ, Yoon HK, Kang SG, Bok KN, Jung KY, Kim L, Lee EI. 2016. Exposure to dim artificial light at night increases REM sleep and awakenings in humans. Chronobiol Int. 33(1):117-123.

Cho CH, Yoon HK, Kang SG, Kim L, Lee EI, Lee HJ. 2018. Impact of exposure to dim light at night on sleep in female and comparison with male subjects. Psychiatry Investig. 15(5):520530.

Cohen D, Atun-Einy O, Scher A. 2012. Seasonal effect on infants' sleep regulation: A preliminary study in a mediterranean climate. Chronobiol Int. 29(10):1352-1357.

Duenas-Meza E, Bazurto-Zapata MA, Gozal D, González-García M, Durán-Cantolla J, TorresDuque CA. 2015. Overnight polysomnographic characteristics and oxygen saturation of healthy infants, 1 to 18 months of age, born and residing at high altitude (2,640 meters). Chest. 148(1):120-127.

Eaton-Evans J, Dugdale AE. 1988. Sleep patterns of infants in the first year of life. Arch Dis Child. 63:647-649.

Galland BC, Taylor BJ, Elder DE, Herbison P. 2012. Normal sleep patterns in infants and children: A systematic review of observational studies. Sleep Med Rev. 16(3):213-222. 
Goodwin JL, Enright PL, Kaemingk KL, Rosen GM, Morgan WJ, Fregosi RF, Quan SF. 2001. Feasibility of using unattended polysomnography in children for research--report of the tucson children's assessment of sleep apnea study (TuCASA). Sleep. 24(8):937-944.

Gooley JJ, Chamberlain K, Smith KA, Khalsa SB, Rajaratnam SM, Van Reen E, Zeitzer JM, Czeisler CA, Lockley SW. 2011. Exposure to room light before bedtime suppresses melatonin onset and shortens melatonin duration in humans. J Clin Endocrinol Metab. 96(3):463-472.

Harrison Y. 2004. The relationship between daytime exposure to light and night-time sleep in 6-12week-old infants. J Sleep Res. 13(4):345-352.

Higuchi S, Nagafuchi Y, Lee SI, Harada T. 2014. Influence of light at night on melatonin suppression in children. J Clin Endocrinol Metab. 99(9):3298-3303.

Iglowstein I, Jenni OG, Molinari L, Largo RH. 2003. Sleep duration from infancy to adolescence: Reference values and generational trends. Pediatrics. 111(2):302-307.

Iwata S, Fujita F, Kinoshita M, Unno M, Horinouchi T, Morokuma S, Iwata O. 2017. Dependence of nighttime sleep duration in one-month-old infants on alterations in natural and artificial photoperiod. Sci Rep. 2017; 7(1):44749. doi:10.1038/srep44749

Jenni OG, Borbely AA, Achermann P. 2004. Development of the nocturnal sleep electroencephalogram in human infants. Am J Physiol. 286(3):528-538.

Kärki A, Paavonen EJ, Satomaa A, Saarenpää-Heikkilä O, Huhtala H, Himanen S. 2019. Sleep architecture is related to birth season in 1-month-old infants. Chronobiol Int. 36(9):1217-1226.

Landis JR, Koch GG. 1977. An application of hierarchical kappa-type statistics in the assessment of majority agreement among multiple observers. Biometrics. 33(2):363-374. 
Leu R., Beyderman L, Botzolakis E, Surdyka K, Wang L, Malow B. 2011. Relation of melatonin to sleep architecture in children with autism. J Autism Dev Disord. 41(4): 427-433.

Louis J, Cannard C, Bastuji H, Challamel MJ. 1997. Sleep ontogenesis revisited: A longitudinal 24hour home polygraphic study on 15 normal infants during the first two years of life. Sleep. 20(5):323-333.

Montemitro E, Franco P, Scaillet S, Kato I, Groswasser J, Villa MP, Kahn A, Sastre J, Ecochard R, Thiriez G, et al. 2008. Maturation of spontaneous arousals in healthy infants. Sleep. 31(1):4754.

O'Connell SE, Griffiths PL, Clemes SA. 2014. Seasonal variation in physical activity, sedentary behaviour and sleep in a sample of UK adults. Ann Hum Biol. 41(1):1-8.

Paakkonen T, Leppaluoto J, Makinen TM, Rintamaki H, Ruokonen A, Hassi J, Palinkas LA. 2008. Seasonal levels of melatonin, thyroid hormones, mood, and cognition near the arctic circle. Aviat Space Environ Med. 79(7):695-699.

Paavonen EJ, Morales-Muñoz I, Pölkki P, Paunio T, Porkka-Heiskanen T, Kylliäinen A, Partonen T, Saarenpää-Heikkilä O. 2019. Development of sleep-wake rhythms during the first year of age. J Sleep Res. 2019; 00:e12918. doi: 10.1111/jsr.12918.

Paavonen EJ, Saarenpaa-Heikkila O, Polkki P, Kylliainen A, Porkka-Heiskanen T, Paunio T. 2017. Maternal and paternal sleep during pregnancy in the child-sleep birth cohort. Sleep Med. 29:4756.

Pattyn N, Mairesse O, Cortoos A, Marcoen N, Neyt X, Meeusen R. 2017. Sleep during an antarctic summer expedition: New light on “polar insomnia”. J Appl Physiol. 122(4):788-794. 
Pattyn N, Van Puyvelde M, Fernandez-Tellez H, Roelands B, Mairesse O. 2018. From the midnight sun to the longest night: Sleep in antarctica. Sleep Med Rev. 37:159-172.

Paul MA, Love RJ, Hawton A, Arendt J. 2015. Sleep and the endogenous melatonin rhythm of high arctic residents during the summer and winter. Physiol Behav. 141:199-206.

Portaluppi F, Smolensky MH, Touitou Y. 2010. Ethics and methods for biological rhythm research on animals and human beings. Chronobiol Int. 27(9-10):1911-1929.

Rohr KE, Pancholi H, Haider S, Karow C, Modert D, Raddatz NJ, Evans J. 2019. Seasonal plasticity in GABAa signaling is necessary for restoring phase synchrony in the master circadian clock network. eLife. 2019;8:e49578. doi:10.7554/eLife.49578

Sadeh A, Mindell JA, Luedtke K, Wiegand B. 2009. Sleep and sleep ecology in the first 3 years: A web-based study. J Sleep Res. 18(1):60-73.

Satomaa A, Saarenpää-Heikkilä O, Paavonen EJ, Himanen S. 2015. The adapted american academy of sleep medicine sleep scoring criteria in one month old infants: A means to improve comparability? Clin Neurophysiol. 127(2):1410-1418.

Sivan Y, Laudon M, Tauman R, Zisapel N. 2001. Melatonin production in healthy infants: Evidence for seasonal variations. Pediatr Res. 49(1):63-68.

Teng A, Bartle A, Sadeh A, Mindell J. 2012. Infant and toddler sleep in Australia and New Zealand. J Paediat Child Health. 48(3):268-273.

Time and Date AS. 2019. Tampere, Finland - Sunrise, sunset, and daylenght. Stavanger (Norway): Time and Date AS; [Accessed 2019 Nov 16,]. Available from: https://www.timeanddate.com/sun/finland/tampere 
Touchette E, Dionne G, Forget-Dubois N, Petit D, Pérusse D, Falissard B, Tremblay RE, Boivin M, Montplaisir JY. 2013. Genetic and environmental influences on daytime and nighttime sleep duration in early childhood. Pediatrics. 2013;131(6):e1874-80. doi:10.1542/peds.2012-2284.

Wams EJ, Woelders T, Marring I, Van Rosmalen L, Beersma DGM, Gordijn MCM, Hut RA. 2017. Linking Light Exposure and Subsequent Sleep: A Field Polysomnography Study in Humans. Sleep. 2017;40(12):zxs165. doi:10.1093/sleep/zsx165.

Yetish G, Kaplan H, Gurven M, Wood B, Pontzer H, Manger PR, Wilson C, McGregor R, Siegel JM. 2015. Natural sleep and its seasonal variations in three pre-industrial societies. Curr Biol. 25(21):2862-2868. 


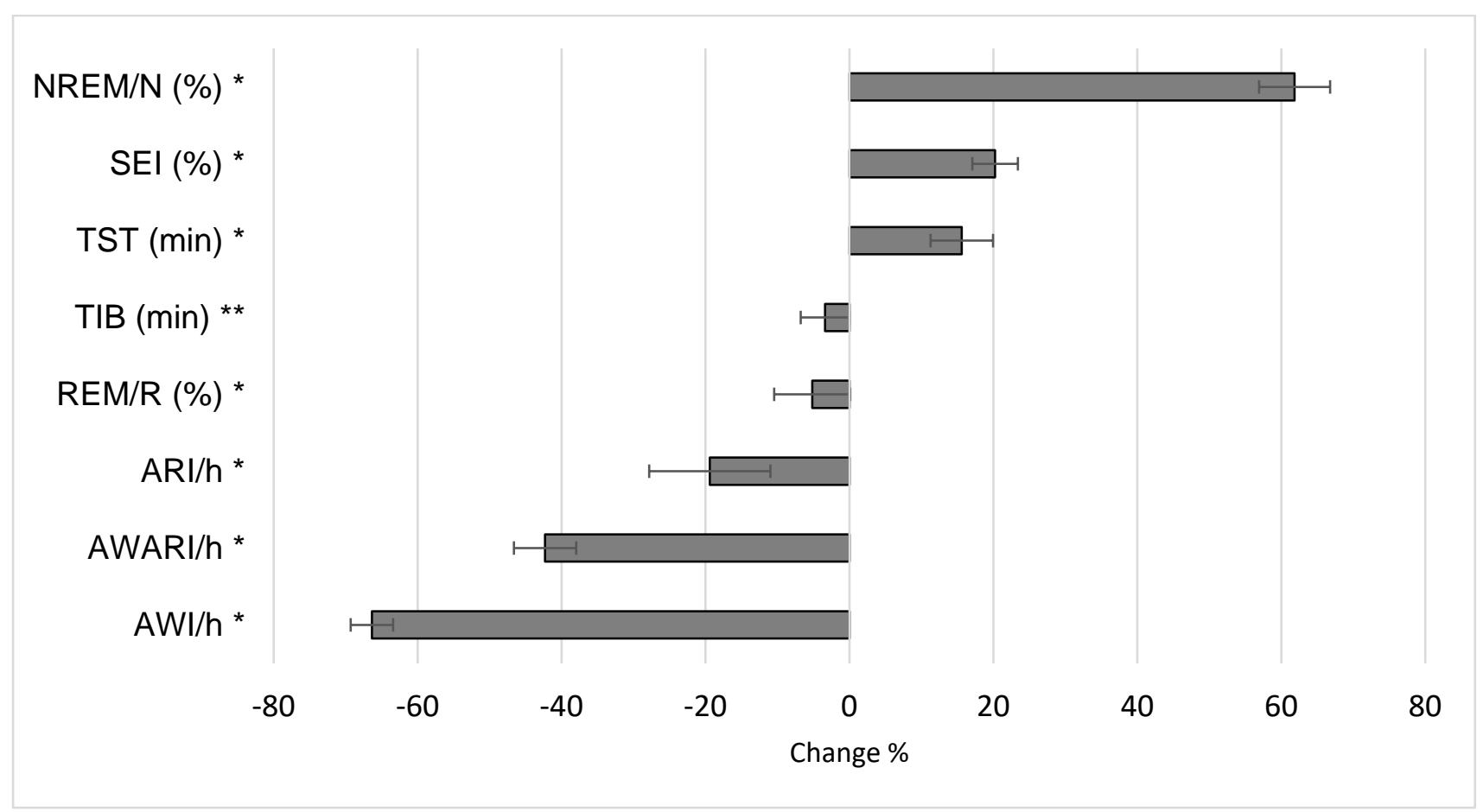

Figure 1: Change\% of sleep parameters in 69 infants between 1 month and 8 month PSG with $95 \%$ confidence intervals.

NREM/N (\%) = percentage of NREM and N-sleep of TST; SEI (\%) = percentage of SEI\% (TST/TIB); TST = total sleep time (in minutes); TIB = time in bed (in minutes); REM/R (\%) = percentage of REM and R-sleep of TST; ARI/h = arousal index/h; AWARI/h = sum of arousal and awakening indexes/h; AWI/h = awakening index/h.

* p-value $\leq 0.001$

** p-value 0.020 
Table 1. Sleep parameters and demographic data of 8-month-old infants $(n=72)$

\begin{tabular}{|c|c|c|}
\hline Parameter $^{1}$ & Average $^{2}$ & Variation $^{3}$ \\
\hline Age (months) & 7.8 & $7.7,8.1$ \\
\hline Girls/Boys (n) & $38 / 34$ & \\
\hline Naps/d (n) & $2 / 45 / 18$ & \\
\hline Weight (g) & 8511.8 & 126.1 \\
\hline Time in bed (min) & 696.6 & 62.7 \\
\hline Total sleep time (min) & 548.5 & 54.1 \\
\hline NREM (\%) & 66.1 & 5.3 \\
\hline N1 (\%) & 6.5 & 3.4 \\
\hline N2 (\%) & 25.5 & 6.9 \\
\hline N3 (\%) & 34.1 & 8.8 \\
\hline REM (\%) & 33.7 & 5.1 \\
\hline SEI (\%) & 79.0 & 7.3 \\
\hline WASO (min) & 117.2 & 49.7 \\
\hline Sleep onset latency (min) & 27.0 & $9.4,44.8$ \\
\hline REM-lat (min) & 50.3 & 27.4 \\
\hline Awakening index/h & 2.8 & 0.8 \\
\hline Arousal index/h & 7.9 & 2.6 \\
\hline AWARI/h & 10.7 & 2.6 \\
\hline
\end{tabular}

${ }^{1}$ Naps/d (n) =Number of naps between 08:00h and 20:00h/day (1/2/3 or more). NREM, N1, N2, N3, REM (\%) = corresponding sleep stage percentages of TST. SEI \%= Sleep efficiency index (TST/TIB x 100). WASO = wake after sleep onset (minutes). REM lat = REM onset latency (minutes). AWARI/h = Sum of awakenings and arousals/hour.

${ }^{2}$ With Gaussian variables, mean is presented; with non-Gaussian variables median. With categorical variables frequencies are presented.

${ }^{3}$ With Gaussian variables, SD is presented; with non-Gaussian variables, quartiles (Q1, Q3) are presented. 
Table 2. Sleep parameters and demographic data of 8-month-old infants $(n=72)$ according to birth seasons.

\begin{tabular}{|c|c|c|c|c|c|c|c|c|c|c|}
\hline \multirow[b]{2}{*}{ Parameter ${ }^{1}$} & \multicolumn{2}{|c|}{$\begin{array}{l}\text { Increasing lightness } \\
\text { (IL, Feb 5-May 5) } \\
\quad(\mathrm{n}=11)\end{array}$} & \multicolumn{2}{|c|}{$\begin{array}{c}\text { Light } \\
(\mathrm{L}, \text { May 6-Aug 5) } \\
(\mathrm{n}=11)\end{array}$} & \multicolumn{2}{|c|}{$\begin{array}{l}\text { Increasing darkness } \\
\text { (ID, Aug 6-Nov 6) } \\
\quad(\mathrm{n}=36)\end{array}$} & \multicolumn{2}{|c|}{$\begin{array}{c}\text { Dark } \\
(\mathrm{D}, \text { Nov 7-Feb 4) } \\
(\mathrm{n}=14)\end{array}$} & \multirow[b]{2}{*}{ p-value } & \multirow[b]{2}{*}{$\begin{array}{l}\text { Adjusted }{ }^{4} \\
\text { p-value }\end{array}$} \\
\hline & Average $^{2}$ & Variation ${ }^{3}$ & Average $^{2}$ & Variation $^{3}$ & Average $^{2}$ & Variation $^{3}$ & Average $^{2}$ & Variation $^{3}$ & & \\
\hline Age (months) & 7.7 & $7.7,7.9$ & 8.0 & $7.9,8.0$ & 7.7 & $7.6,8.0$ & 8.4 & $7.7,8.8$ & 0.007 & \\
\hline Girls/Boys (n) & $7 / 4$ & & $8 / 3$ & & $18 / 18$ & & $5 / 9$ & & 0.276 & \\
\hline Naps/d (n) & $0 / 8 / 2$ & & $1 / 4 / 5$ & & $0 / 24 / 8$ & & $1 / 9 / 3$ & & 0.251 & \\
\hline Weight (g) & 8221.0 & 1225.1 & 8885.0 & 947.4 & 8435.9 & 845.5 & 8636.8 & 1101.7 & 0.449 & \\
\hline Time in bed (min) & 698.2 & 71.7 & 709.1 & 48.9 & 696.1 & 59.5 & 686.8 & 77.0 & 0.858 & \\
\hline TST (min) & 546.6 & 60.7 & 551.2 & 35.6 & 551.6 & 57.8 & 540.0 & 55.4 & 0.923 & \\
\hline NREM (\%) & 63.6 & $61.0,65.5$ & 64.5 & $61.2,70.1$ & 68.2 & $64.8,69.6$ & 67.7 & $62.9,71.0$ & 0.323 & \\
\hline N1 (\%) & 5.9 & $4.0,6.9$ & 6.2 & $3.1,8.5$ & 6.9 & $4.2,8.5$ & 4.3 & $3.7,6.8$ & 0.419 & \\
\hline N2 (\%) & 24.6 & 5.1 & $29.3 *$ & 5.6 & 26.2 & 7.6 & $21.6^{*}$ & 5.7 & 0.035 & 0.135 \\
\hline N3 (\%) & 33.4 & 6.0 & $29.4^{*}$ & 6.7 & 33.6 & 8.9 & $39.5^{*}$ & 9.7 & 0.028 & 0.104 \\
\hline REM (\%) & 36.4 & $34.1,39.0$ & 35.5 & $29.9,38.8$ & 31.9 & $30.4,35.2$ & 32.4 & $29.0,37.0$ & 0.278 & \\
\hline SEI (\%) & 79.0 & 74.5, 81.6 & 77.9 & $75.1,81.1$ & 79.5 & $75.8,85.2$ & 81.8 & $76.2,86.6$ & 0.716 & \\
\hline WASO (min) & 109.0 & $69.5,122.0$ & 123.5 & $97.5,153.5$ & 105.5 & $85.4,131.3$ & 116.0 & $75.6,157.0$ & 0.597 & \\
\hline Sleep lat (min) & 39.5 & $30.0,54.0$ & 25.0 & $6.5,42.5$ & 28.5 & $12.4,51.8$ & 14.5 & $6.8,27.4$ & $4 \quad 0.074$ & \\
\hline REM lat (min) & 48.0 & $33.5,54.4$ & 42.3 & $36.3,54.9$ & 43.5 & $35.1,54.6$ & 56.3 & $41.0,71.8$ & 0.088 & \\
\hline AWI/h & 2.7 & 1.1 & 2.8 & 0.8 & 2.7 & 0.7 & 2.9 & 1.0 & 0.867 & \\
\hline $\mathrm{ARI} / \mathrm{h}$ & 6.8 & 2.1 & 8.6 & 2.8 & 8.4 & 2.5 & 7.0 & 2.6 & 0.125 & \\
\hline AWARI/h & 9.5 & 2.5 & 11.4 & 3.3 & 11.1 & 2.3 & 10.0 & 2.6 & 0.174 & \\
\hline
\end{tabular}


Table 3. Sleep parameters and demographic data of 8-month-old infants $(\mathrm{n}=72)$ according to PSG seasons.

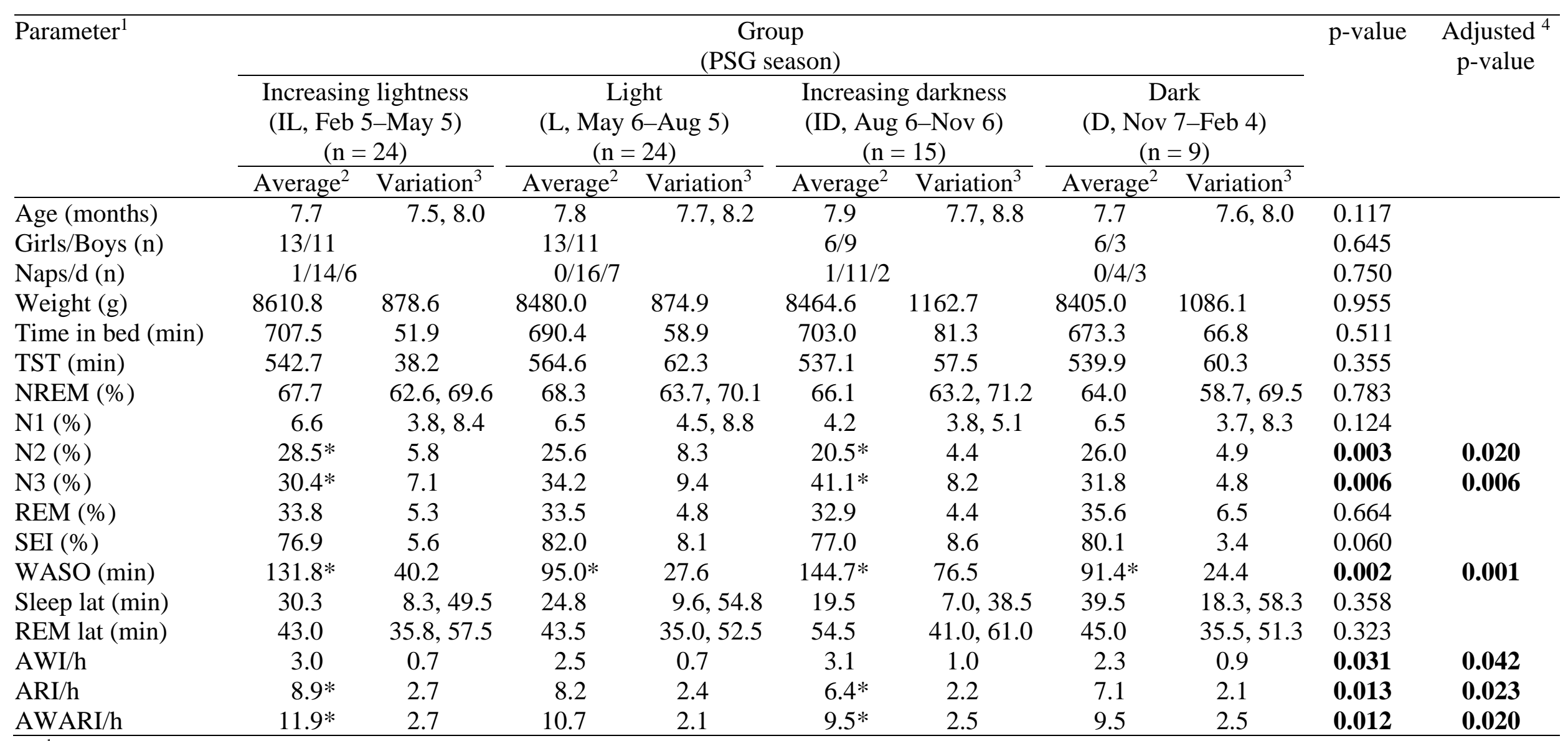

${ }^{1} \mathrm{Naps} / \mathrm{d}(\mathrm{n})=$ Number of naps between 08:00h. and 20:00h/day (1/2/3 or more). TST = Total sleep time (minutes). NREM, N1, N2, N3, REM (\%) = corresponding percentages of sleep stages of TST. SEI \% = Sleep efficiency index (TST/TIB x 100). WASO = Wake after sleep onset (minutes). Sleep lat = Sleep onset latency (minutes). $\mathrm{REM}$ lat = REM onset latency (minutes). AWI/h = Awakening index/hour. ARI/h = Arousal index/hour. AWARI/h = Sum of awakenings and arousals/hour.

${ }^{2}$ With Gaussian variables, mean is presented; with non-Gaussian variables, median is presented. With categorical variables frequencies are presented.

${ }^{3}$ With Gaussian variables, SD is represented; with non-Gaussian variables, quartiles (Q1, Q3).

${ }^{4}$ Adjusted with age and sex.

${ }^{*}$ Post-hoc tests showed significant differences between groups as follows: N2\%: IL vs. ID ( $p=0.001$ ); N3\%: IL vs. ID ( $p=0.003$ ); WASO: IL vs L ( $p=0.034$ ) and L vs ID ( $\mathrm{p}=0.008)$, and ID vs $\mathrm{D}(\mathrm{p}=0.035) ; \mathrm{ARI} / \mathrm{h}$ : IL vs ID ( $\mathrm{p}=0.011)$; AWARI/h: IL vs ID ( $\mathrm{p}=0.019)$. 